\title{
APORTACIONES A LA AFIDOFAUNA ÍBERO-BALEAR (HEMIPTERA, APHIDIDAE) A PARTIR DE LAS FOTOGRAFÍAS (Y DE SUS METADA- TOS) DEPOSITADAS EN EL "BANCO TAXONÓMICO FAUNÍSTICO DIGITAL DE LOS INVERTEBRADOS IBÉRICOS” (B.T.F.D.I.I.)*
}

\author{
N. Pérez Hidalgo ${ }^{1}$ Á. Umaran², M. P. Mier Durante ${ }^{1} \&$ J. M. Nieto Nafría ${ }^{1}$
}

\section{RESUMEN}

En el contexto de los estudios taxonómicos y faunísticos de pulgones del proyecto Fauna Ibérica, analizamos: 1) el grado de fiabilidad que se puede alcanzar en la identificación de las fotografías de pulgones depositadas en el Banco Taxonómico Faunístico Digital de los Invertebrados Ibéricos (B.T.F.D.I.I.), 2) la utilidad de la información faunística y las relaciones planta-pulgón; y 3) los posibles sesgos en cuanto a las especies fotografiadas.

Se han revisado 443 fotografías de pulgones o áfidos realizadas por 53 fotógrafos y depositadas hasta abril de 2009 en la carpeta Aphididae del B.T.F.D.I.I. La identificación se ha realizado exclusivamente "online" y de las 443 fotografías analizadas, 277 (el $62,5 \%$ ) se han identificado hasta especie y 166 (el 37,5\%) hasta género o familia. Se han identificado 65 especies, cuyos datos permiten que 28 especies amplíen el área de distribución conocida, en una o más provincias en la península Ibérica. Además se han establecido seis nuevas relaciones planta hospedadora-pulgón en nuestros territorios. La mayoría de las fotografías se realizan a especies polífagas y ampliamente distribuidas, a especies que presentan llamativas coloraciones y/o gran tamaño y a las malformaciones (agallas y pseudoagallas) que inducen en sus hospedadores.

Palabras clave: Pulgones, Áfidos, Aphididae, Península Ibérica.

\section{ABSTRACT \\ Contributions to the ibero-balear aphid fauna (Hemiptera, Aphididae) from photos (and their metadata) deposited in the "Banco Taxonómico Faunístico Digital de los Invertebrados Ibéricos" (B.T.D.I.I.)}

In the context of the "Fauna Ibérica Project" we analyzed: 1) the trustworthiness in the identification from pictures of aphids deposited in the "Banco Taxonómico Faunístico Digital de los Invertebrados Ibéricos (B.T.F.D.I.I.)", 2) the utility of the faunistic information and the host plant-aphid relations; and 3) the possible slants in relation to the species photographed.

443 pictures of aphids realized for 53 photographers and deposited in the BT.F.D.I.I until April 2009 have been checked and the identification has been realized exclusively "online". 277 (the 62.5\%) have been identified until species and 166 (the 37.5\%) until

Departamento de Biodiversidad y Gestión Ambiental, Universidad de León. 24071. León, España; E-mail: nperh@unileon.es /mpmied@unileon.es / jmnien@unileon.es

C./ Hilados, 1 esc. Izq. 3D. Torrejón de Ardoz. 28850 Madrid (España) E-mail: aumaran@gmail.com

* Trabajo elaborado en el contexto del Proyecto Fauna Ibérica IX (CGL2007-66786-C08-03). 
genera o family. In total 65 species have been identified and 28 species increased its known distribution area in the Iberian Peninsula. Moreover, six new host-plant-aphid relations have been established. The mayor pictures are making to polyphagous and common species, to species that present loud colorations and/or great size and to species that induced malformations (galls or pseudogalls) in its hosts.

Key Words: Pulgones, Áfidos, Aphididae, Península Ibérica.

\section{Introducción}

El creciente interés social por la naturaleza que se da en los países desarrollados, ha hecho que numerosos aficionados a la fotografía dediquen su tiempo a fotografiar insectos y otros pequeños animales. La calidad de las imágenes que captan ha aumentado considerablemente en los últimos tiempos, debido a la mejora de los medios técnicos de que se dispone. Al mismo tiempo, Internet ha contribuido a difundir este tipo de actividades y sobre todo a facilitar que exista un punto de encuentro entre aficionados a la fotografía y especialistas taxónomos, sean aficionados o profesionales (Carling \& Harrison, 1996; Esteban et al., 1997).

La plataforma "Biodiversidad Virtual" (www.biodiversidadvirtual.com) viene elaborando desde 2008 una base de datos utilizando la fotografía digital para, entre otras cosas, formar el "Banco Taxonómico Faunístico Digital de los Invertebrados Ibéricos (B.T.F.D.I.I.)" (http://www.insectariumvirtual.com) que es la sección de invertebrados del proyecto, una plataforma cuyos fines son el conocimiento, la divulgación y la conservación de la biodiversidad a través de la participación ciudadana. Ésta está abierta a estudios científicos tanto de profesionales como de aficionados; pero, hasta la fecha, pocos han sido los trabajos (investigaciones y/o publicaciones) que han utilizado los datos existentes.

Con el fin de evaluar la utilidad de estas nuevas formas de "captura fotográfica", en el contexto de los estudios taxonómicos y faunísticos de pulgones que se vienen desarrollando en el proyecto Fauna Ibérica, analizamos: 1) el grado de fiabilidad que se puede alcanzar en la identificación de las fotografías de pulgones depositadas en el B.T.F.D.I.I., 2) la utilidad de la información que de ellas se deriva en cuanto a la ampliación de la distribución provincial y a las relaciones planta-pulgón; y 3) los posibles sesgos en cuanto a las especies fotografiadas.

\section{Material y métodos}

Hemos revisado las 443 fotografías de pulgones o áfidos depositadas hasta abril de 2009 en la car- peta "Aphididae" del B.T.F.D.I.I. que han sido realizadas por 53 fotógrafos.

La identificación se ha efectuado exclusivamente "online" utilizando la información disponible en los volúmenes $(11,19$ y 28$)$ dedicados a pulgones en el proyecto Fauna Ibérica (Nieto Nafría \& Mier Durante, 1998; Nieto Nafría et al., 2003, 2005), en las claves de alados de la región mediterránea (Remaudière \& Seco Fernández, 1990) y en las monografías de Blackman e Eastop dedicadas a los áfidos de las plantas arbóreas (1994), cultivadas (2000) y herbáceas y arbustivas (2006). Los criterios taxonómicos y nomenclaturales son los mismos que se siguen en los proyectos Fauna Ibérica (http://iberfauna.mncn.csic.es/) y Fauna Europaea (http://www.faunaeur.org/).

Se considera una "muestra fotográfica" la fotografía o grupo de fotografías tomadas por una misma persona en una misma localidad y sobre una misma planta, aunque el autor haya realizado varias fotografías de la misma colonia o fotografías de diversos ejemplares y/o de diversas formas de la misma especie. Los metadatos de las fotografías incluyen información sobre el autor de la fotografía, la fecha de realización, el lugar (incluidas las coordenadas geográficas y la altitud) y la planta hospedadora.

\section{Resultados y discusión}

\section{GRADO DE EXACTITUD EN LAS IDENTIFICACIONES}

Como sucede en la mayoría de los grupos de artrópodos, algunas especies se pueden identificar sin problemas (incluso a nivel específico) simplemente observando el aspecto de la colonia y unos pocos caracteres diagnósticos (Mier-Durante \& Pérez-Hidalgo, 2004; Pérez Hidalgo, 2007). Sin embargo, en otros casos es necesario observar los caracteres morfológicos con un mayor detalle e incluso realizar preparaciones microscópicas, lo cual no ha sido posible en este caso.

De las 443 fotografías, 277 (el 62,5\%) se han identificado hasta especie y 166 (el 37,5\%) hasta género o incluso sólo hasta familia. Sin embargo, hay que destacar que este porcentaje podría aumentar si en los metadatos de muchas fotografías se 


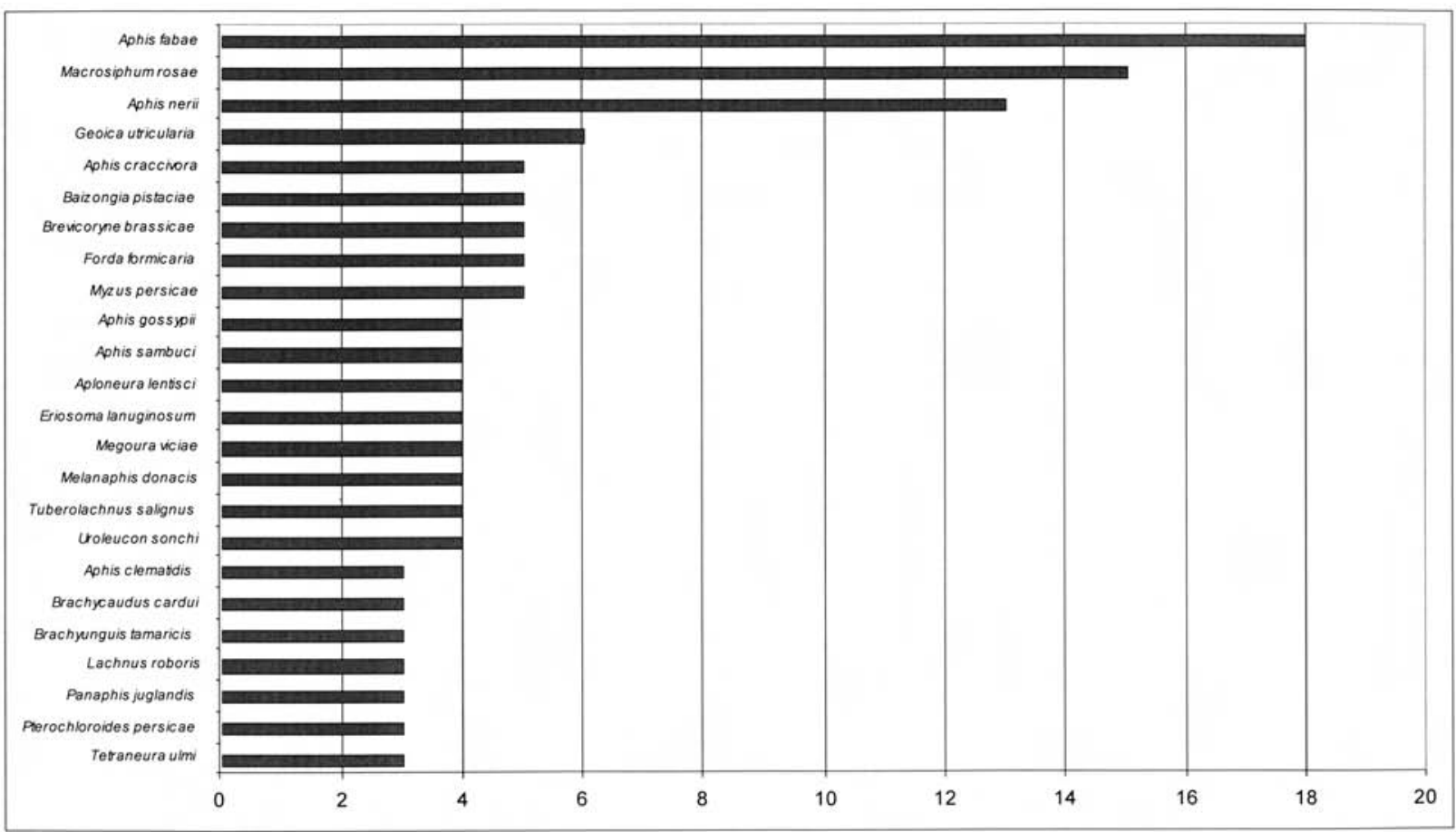

Fig. 1.- Especies más fotografiadas (con más de tres "muestras fotográficas") depositadas en el "Banco Taxonómico Faunístico Digital de los Invertebrados Ibéricos" (B.T.F.D.I.I.) hasta abril de 2009. Las fotografías de las especies de eriosomatinos (G. utricularia, B. pistaciae, F. formicaria, A. lentisci, E. lanuginosum y T. ulmi) son de las agallas que inducen en sus hospedadores primarios.

Fig. 1.- More photographed species (with more than three "photographic samples") deposited in the "Banco Taxonómico Faunístico Digital de los Invertebrados Ibéricos" (B.T.F.D.I.I.) until April 2009. The photos of eriosomatids (G. utricularia, B. pistaciae, F. formicaria, A. lentisci, E. lanuginosum y T. ulmi) are of the galls that they induce in their primary hosts.

hubiese aportado la identidad de la planta hospedadora de la colonia fotografiada.

\section{UTILIDAD CIENTÍFICA DE LA INFORMACIÓN}

En total, se han identificado 65 especies (ver Apéndice), lo que ha permitido que 28 especies amplíen el área de distribución conocida, en una o más provincias, actualizando su distribución publicada en los volúmenes 11, 19 y 28 de Fauna Ibérica. En la relación de especies identificadas, se marca con un asterisco $(*)$ la novedad provincial y con dos $(* *)$ la nueva relación planta-pulgón para la península Ibérica.

Dos especies, consideradas invasoras, merecen ser destacadas ya que presentan pocas citas en la Península: Pterochloroides persicae y Neotoxoptera violae. Pterochloroides persicae es una especie monoica sobre especies de Prunus (melocotoneros y albaricoqueros principalmente), aunque se ha citado también sobre pomoideas y excepcionalmente sobre Citrus sp. y Ficus carica. Su área de distribución originalmente comprendía desde la parte oriental de la cuenca mediterránea hasta la India, pero en los últimos 20 años se ha ido extendiendo hacia occidente (Nieto Nafría et al., 2003). La especie sólo se había citado sobre almendros en la región de Murcia (Hermoso de Mendoza \& Lacasa, 1995; Lucas Espadas, 1995) y ahora las fotografías de esta especie sobre un melocotonero en Alicante (Villena) y en un limonero en Madrid (Leganés), amplían considerablemente hacia el Oeste y ligeramente hacia el Noreste el área de distribución conocida de esta especie en la Península, que seguro es más amplia ya en nuestros territorios.

La cita de Neotoxoptera violae es la segunda en España. Previamente era conocida (Meliá, 1991) por una muestra sobre Viola odorata y alados capturados en trampa en Castellón. $N$. violae vive sobre especies del género Viola y se encuentra ampliamente extendida en Norteamérica, lugar del 


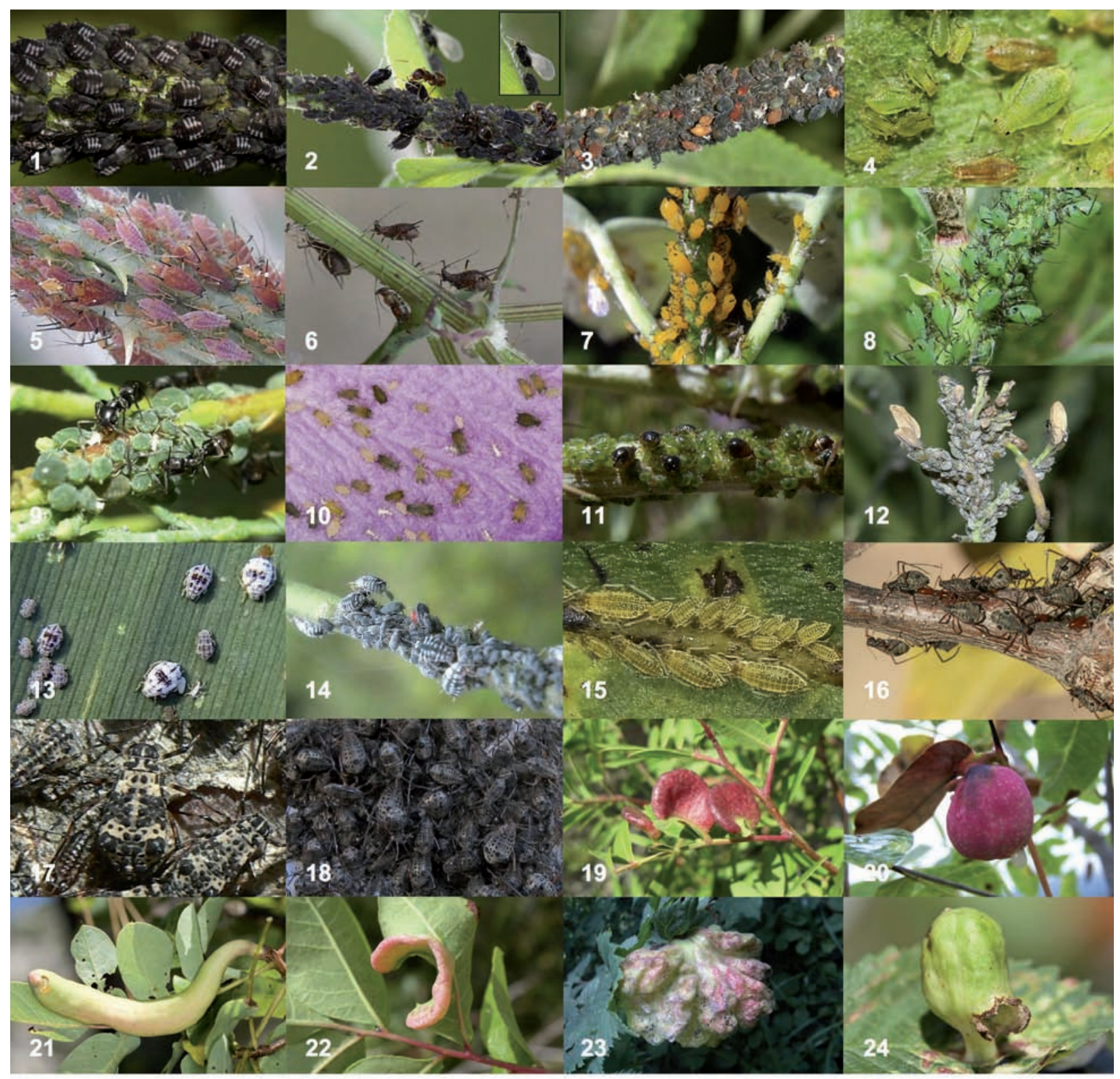

Fig. 2.- Colonias de Aphis fabae (1), Aphis craccivora (2) [Fotos de José Manuel Sesma], Aphis sambuci (3) [Foto de Luis M. Lafuente], Myzus persicae (4) [Foto de Javier Aznar], Macrosiphum rosae (5) [Foto de Miguel Yuste], Uroleucon sonchi (6) [Foto de Fani Martínez], Aphis nerii (7) [Foto de Josep María Solé], Megoura viciae (8) [Foto de David Molina], Brachyunguis tamaricis (9) [Foto de Jordi Clavell], Aphis gossypii (10) [Foto de Ángel Umaran], Brachycaudus cardui (11) [Foto de Jaime Díaz], Brevicoryne brassicae (12) [Foto de Luis M. Lafuente], Melanaphis donacis (13) [Foto de Isidro Martínez], Aphis clematidis (14) [Foto de Luis Fernández], Panaphis juglandis (15) [Foto de Carlos Castañeda], Lachnus roboris (16) [Foto de Javier Cambra], Pterochloroides persicae (16) [Foto de David Molina], Tuberolachnus salignus (18) [Foto de Juan Carlos Cambronero]; y agallas de Aploneura lentisci (19) [Foto de Constantino Escuer], Geoica utricularia (20) [Foto de David Molina], Baizongia pistaciae (21) [Foto de Ángel Umaran], Forda formicaria (22) [Foto de Nicolás Pérez], Eriosoma lanuginosum (23) [Foto de Belén Amarante] y Tetraneura ulmi (24) [Foto de Ángel Umaran]. 
que puede ser originaria, además de haber sido citada también en Sudamérica, Hawai y Corea; e introducida en Europa: Italia (Barbagallo \& Cocuzza, 1998) y Francia (Germain \& Deogratias, 2008).

\section{Posibles SESGOS EN CUANTO A LAS ESPECIES FOTO- GRAFIADAS}

Considerando únicamente las fotografías que hemos podido identificar hasta especie, se observa que la mayoría de ellas (fig. 1) se realizan a especies muy comunes y/o polífagas (figs. 2, fotos 1-6) destacando Aphis fabae o Myzus persicae. Un segundo grupo son los pulgones que presentan llamativas coloraciones (fig. 2, fotos 7-11), como en el caso de Aphis nerii, Macrosiphum rosae y Megoura viciae, o que están cubiertas en mayor o menor medida de cera (fig 2, fotos 12-14) como Melanaphis donacis, Brevicoryne brassicae y Aphis clematidis. Un tercer grupo son las especies de gran tamaño (fig 2, fotos 15-18), entre las que destacan Tuberolachnus salignus, Lachnus roboris y Pterochloroides persicae. Además hay que considerar que estas especies suelen presentar colonias muy abundantes en plantas que frecuentemente se usan como ornamentales en parques y jardines, hecho éste que contribuye a aumentar su visibilidad.

Mención aparte merecen las especies de eriosomatinos (Eriosomatinae) que inducen llamativas malformaciones en forma de agallas o pseudoagallas en sus hospedadores (fig 2, fotos 19-24), principalmente en olmos (Eriosoma lanuginosum, Tetraneura ulmi), cornicabras (Baizongia pistaciae, Geoica utricularia o Forda formicaria) y chopos negros (Pemphigus immunis), y que suelen ser más fácilmente visibles que las colonias de los pulgones de las restantes subfamilias.

\section{AGRADECIMIENTOS}

Nuestro agradecimiento a los autores de las fotografías depositadas en el Banco taxonómico, sin cuya curiosidad por los pequeños animales este trabajo no habría podido realizarse:
Aitor Valdeón, Antonio García, Antonio J. Muñoz, Antonio Verdugo, Arturo López Gallego, Beatriz Etxeberria, Belén Amarante, Blas Fernández, Carlos Castañeda Navarro, Carlos Galeano, Constantino Escuer, Daniel Gutiérrez, David Molina, Divina Aparicio de Andrés, Fani Martínez, Ferrán Turmo Gort, Guillermo Booth Rea, Isidro Martínez, Jacinto Benhadi Marín, Jaime Díaz, Javier Aznar, Javier Cambra Mur, Javier Gállego, Joaquín Portela, Jordi Clavell, Jordi Moratal, José Luis Palacios López, José Manuel Barrera, José Manuel Sesma, José Ramón Correas, Josep María Solé, Juan Antonio Perea, Juan Carlos Cambronero, Klaus Kamppeter, Laura Collado, Laura Comellas, Lola López, Luis Fernández, Luis M. Lafuente, Max Delgado Corteguera, Miguel Ángel Gómez de Dios, Miguel Romá Sáez, Miguel Yuste De Santos, Nacho Cabellos, Paco Alarcón, Paqui Mars, Rafael López Loureiro, Ricard Senent, Román Rojo Olivas, Sue Eatock, Tony Romero y Xavier Sanjuán.

Agradecimiento que se extiende a Antonio Ordóñez, José Manuel Sesma, Jordi Clavell y Juan Carlos Cambronero, creadores del proyecto Biodiversidad Virtual e impulsores de este trabajo y otros similares, y que además han realizado aportaciones al mismo.

\section{Referencias}

Barbagallo, S. \& Cocuzza, G. E., 1998. Sulla presen$\mathrm{za}$ in Sicilia dell'afide Neotoxoptera violae (Pergande). Bolletino di Zoologia agraria e di Bachicoltura, Ser. II, 30(2): 321-326.

Blackman, R. L. \& EAstop, V. F., 1994. Aphids on the World's Trees. An Identification and Information Guide. CAB International. Wallingford. $8+987$ pp. $+16 \mathrm{pl}$.

Blackman, R. L. \& EAStop, V. F., 2000. Aphids on the World's Crops. An identification guide (second edition). J. Wiley \& Sons. Chichester. $8+466$ pp.

Blackman, R. L. \& Eastop, V. F., 2006. Aphids on the World's Herbaceous Plants and Shrubs. (Volume 1 Host Lists and Keys / Volume 2 The aphids). J. Wiley \& Sons. Chichester. $8+1439$ pp.

CARling, R. C. J. \& Harrison, J., 1996. Biodiversity information on the Internet: cornucopia or confusion? Biodiversity Letters, 3: 125-135.

Esteban, M., Bello, E. \& García-Valdecasas, A., 1997. La Zoología en Internet. Graellsia, 53: 3-27.

Fig. 2.- Colonies of Aphis fabae (1), Aphis craccivora (2) [photos by José Manuel Sesma], Aphis sambuci (3) [Photo by Luis M. Lafuente], Myzus persicae (4) [Photo by Javier Aznar], Macrosiphum rosae (5) [Photo by Miguel Yuste], Uroleucon sonchi (6) [Photo by Fani Martínez], Aphis nerii (7) [Photo by Josep María Solé], Megoura viciae (8) [Photo by David Molina], Brachyunguis tamaricis (9) [Photo by Jordi Clavell], Aphis gossypii (10) [Photo by Ángel Umaran], Brachycaudus cardui (11) [Photo by Jaime Díaz], Brevicoryne brassicae (12) [Photo by Luis M. Lafuente], Melanaphis donacis (13) [Photo by Isidro Martínez], Aphis clematidis (14) [Photo by Luis Fernández], Panaphis juglandis (15) [Photo by Carlos Castañeda], Lachnus roboris (16) [Photo by Javier Cambra], Pterochloroides persicae (16) [Photo by David Molina], Tuberolachnus salignus (18) [Photo by Juan Carlos Cambronero]; and galls of Aploneura lentisci (19) [Photo by Constantino Escuer], Geoica utricularia (20) [Photo by David Molina], Baizongia pistaciae (21) [Photo by Ángel Umaran], Forda formicaria (22) [Photo by Nicolás Pérez], Eriosoma lanuginosum (23) [Photo by Belén Amarante] and Tetraneura ulmi (24) [Photo by Ángel Umaran]. 
Germain, J. F. \& Deogratias, J. M., 2008. Confirmation de la présence en France du puceron de la violette. PHM-Revue Horticole, 507: 42-44.

Hermoso de Mendoza, A. \& Lacasa, A., 1995. Primera cita en España de Pterochloroides persicae (Cholodkovsky, 1899) (Hom., Aphidinea: Lachnidae). Boletín de la Asociación Española de Entomología, 19(1-2): 313.

LuCAS Espadas, A., 1995. Incidencia de las Plagas y Enfermedades en las Comunidades Autónomas durante 1994 (S.S.V. de Murcia). Phytoma, 67: 5154.

Melí, A., 1991. Presencia en España de Aphis violae Schouteden y Neotoxoptera violae Pergande (Homoptera, Aphididae) sobre Viola odorata L. Boletín de Sanidad Vegetal, Plagas, 17: 537-543.

Mier-Durante, M. P. \& Pérez-Hidalgo, N., 2004. Hemípteros (Subórdenes Sternorrhyncha, Clypeorrhyncha y Archaeorrhyncha). In: Barrientos, J.A. (ed.). Curso práctico de Entomología. Asociación Española de Entomología, Centro Iberoamericano de la Biodiversidad, Universidat Autònoma de Barcelona (Servei de Publicacions). Alicante, Bellaterra: 625-644.

Nieto Nafría, J. M. \& Mier Durante, M. P., 1988. Hemiptera, Aphididae I. In: Ramos M. A. et al. (eds.). Fauna Ibérica, vol. 11. Museo Nacional de Ciencias Naturales. CSIC. Madrid. 424 pp.

Nieto Nafría, J. M., Mier Durante, M. P., Binazzi, A. \& Pérez Hidalgo, N., 2003. Hemiptera, Aphididae II. In: Fauna Ibérica, vol. 19. Ramos, M. A. et al. (eds.). Museo Nacional de Ciencias Naturales. CSIC. Madrid [2002]. $350 \mathrm{pp}$.

Nieto Nafría, J. M., Mier Durante, M. P., García Prieto, F. \& Pérez Hidalgo, N., 2005. Hemiptera, Aphididae III. In: Ramos M. A. et al. (eds.). Fauna Ibérica, vol. 28. Museo Nacional de Ciencias Naturales. CSIC. Madrid. 362 pp.
PéRez HidAlgo, N., 2007. Algunos consejos para localizar, fotografiar e "intentar" identificar pulgones mediante fotografías. http://www.insectariumvirtual.com/blog-iv/?page_id=224.

Remaudière, G. \& Seco Fernández, M. V., 1990. Claves para ayudar al reconocimiento de alados de pulgones trampeados en la región mediterránea (Hom. Aphidoidea). 2 Vols. Universidad de León (Secretariado de Publicaciones). León. 205 pp. 
Apéndice.- - Listado de especies en orden alfabético y su correspondiente adscripción supragenérica, plantas hospedadoras, provincias, localidades (y parajes), fecha y autor de la fotografía o grupo de fotografías asociadas a la "muestra fotográfica".

Appendix.- List of species in alphabetical order and their respective suprageneric assignation, host-plants, provinces, localities (and places), date and author of the photo or group of photos associated to the "photographic sample".

Aphis clematidis Koch, 1854 (Aphidinae: Aphidini: Aphidina) Clematis flammula:

TARRAGONA*: La Senia, 18-5-2008, Luís Fernández leg.

VALENCIA: Valencia (Jardín Botánico), 28-11-2007, David Molina leg.; Canals, 24-11-2007, Fani Martínez leg.

Aphis craccae Linnaeus, 1758 (Aphidinae: Aphidini: Aphidina) Vicia sp.:

HuEsCA: Benasque, 13-8-2007, Isidro Martínez leg.

Aphis craccivora Koch, 1854 (Aphidinae: Aphidini: Aphidina) Glycyrrhiza glabra:

ZARAGOZA: Zaragoza, 19-8-2007, Isidro Martínez leg.

Melilotus albus:

BARCELONA: Sant Marí d'Albars, 9-8-2008, Jordi Clavell leg.

Gerona: Pardines (Vall de Ribes), 15-7-2007, José Manuel Sesma leg.

Robinia pseudoacacia:

Alicante: Villena (Peña Rubia), 15-6-2008, David Molina leg. Vicia faba:

VALENCIA: Canals, 1-11-2007 y 10-11-2007, Fani Martínez leg.

Aphis fabae Scopoli, 1763 (Aphidinae: Aphidini: Aphidina)

Chenopodium album:

SORIA: Alcozar, 8-7-2007, Divina Aparicio leg.

Chenopodium sp.:

LÉRIDA: Esterri d'Àneu, 30-7-2008, Jordi Clavell leg.

Epipactis sp.:

BARCELONA: Sierra del Garraf, 3-6-2007, Xavier Sanjuan leg.

Foeniculum vulgare:

BARCELONA: Sant Martí d'Albars, 12-8-2008, Jordi Clavell leg.

CASTEllón: Segorbe, 31-10-2007, David Molina leg.

TARRAGONA: Flix, 20-10-2007, David Molina leg.

Nerium oleander:

VAlencia: Betera, 1-5-2008, Miguel Romá leg.

Papaver hybridum:

SORIA: Alcozar, 8-6-2008, Divina Aparicio leg.

Pittosporum tobira:

MADRID: Torrejón de Ardoz, 9-3-2008, Ángel Umaran leg.

VALENCIA: Valencia, 9-10-2007, David Molina leg.

Veratrum album**:

LÉRIDA: Espot, 4-8-2008, Jordi Clavell leg.

Vicia faba:

VALENCIA: Canals, 13-4-2008, Fani Martínez leg.

Vicia sp.:

AlicANTE: Villena (Peña Rubia), 15-6-2008, David Molina leg.

Vinca difformis**.

GeronA: Sant Joan les Fonts, 17-03-2008, Ángel Umaran leg.

Yucca aloifolia:

VAlenCIA: Valencia, 19-10-2007, David Molina leg.

Poaceae:

Gerona: San Pere Pescador, 14-7-2008, Jordi Clavel leg.

Planta hospedadora no identificada:

Asturias: Grado, 18-7-2007, Luis M. Lafuente leg.

Barcelona: La Batllória San Celoni, 22-8-2007, Juan Antonio Perea leg.
LÉRIDA: Esterri d'Àneu, Lérida, 30-7-2008, Jordi Clavell leg.; Espot, 31-7-2008, Jordi Clavell leg.

VALENCIA: Valencia, 5-10-2008, Paqui Mars leg.

Aphis farinosa Gmelin, 1790 (Aphidinae: Aphidini: Aphidina) Salix babylonica:

VALENCIA*: Valencia, 24-10-2007, David Molina leg.

Salix sp.

LA CORUÑA: As Somozas, 31-5-2007, López Loureiro leg.

Aphis gossypii Glover, 1877 (Aphidinae: Aphidini: Aphidina) Cucumis sativus:

MADRID: Madrid, 1-9-2008, Ángel Umaran leg.

Ecballium elaterium:

ZARAGOZA: Juslibol, 9-9-2007, Isidro Martínez leg.

Hibiscus syriacus:

MADRID: Madrid, 1-4-2008, Ángel Umaran leg.

Solanum jasminoides**:

MADRID: Madrid, 30-11-2008, Ángel Umaran leg.

Aphis hederae Kaltenbach, 1843 (Aphidinae: Aphidini: Aphidina) Hedera helix:

MADRID: Madrid, 9-1-2006, Ángel Umaran leg.; 21-2-2008, Miguel Yuste leg.

Aphis idaei van der Goot, 1912 (Aphidinae: Aphidini: Aphidina) Rubus idaeus:

Asturias*: Salas, 17-5-2007, Luis M. Lafuente leg.

Aphis nerii Boyer de Fonscolombe, 1841 (Aphidinae: Aphidini: Aphidina)

Araujia sericifera**:

BArCelona: Parc de Collserola, 19-9-2007, José Manuel Sesma; Esplugues de Llobregat, 10-11-2007, Divina Aparicio leg.

TARRAGONA*: El Catllar, 20-9-2007, Jordi Moratal leg.

Cynanchum acutum:

ZARAGOZA*: Zaragoza, 29-4-2007, Isidro Martínez leg.

Nerium oleander:

BARCELONA: Barcelona, 9-2-2008, José Manuel Sesma leg.

MADRID: Madrid, 5-5-2006, Ángel Umaran leg.

SEvilla: Sevilla, 23-4-2006, Paco Alarcón leg.

TARRAGONA*: Flix, 20-10-2007, David Molina leg.; VandellósL'Almadrava, 11-5-2008, Jordi Clavell leg.

VAlEnCIA: Canals, 4-4-2008, Fani Martínez leg.

Planta hospedadora no identificada:

BADAJOZ*: Almendralejo, 26-5-2006, José Manuel Barrera leg. Barcelona: Castelldefels, 15-6-2007, Juan Antonio Perea leg. GeronA: Empuriabrava, 12-10-2007, Laura Comellas leg.

Aphis ruborum (Börner, 1931) (Aphidinae: Aphidini: Aphidina) Rubus sp.:

BARCELONA: Sant Martí d'Albars, 14-8-2008, Jordi Clavell leg.

Aphis sambuci Linnaeus, 1758 (Aphidinae: Aphidini: Aphidina) Sambucus nigra: 
Asturias: Grado (río Cubia), 14-6-2007, Luis M. Lafuente leg. Barcelona: Berguedà, 5-8-2007, José Manuel Sesma leg.; Sant Martí d'Albars, 21-05-05, Jordi Clavell leg.

GeronA*: Arbucies (Parc del Montseny), 28-10-2007, José Manuel Sesma leg.

Aphis sedi Kaltenbach, 1843 (Aphidinae: Aphidini: Aphidina) Sedum sp.:

LÉRIDA*: Aransa, 21-7-2008, Jordi Clavell leg.

Aphis spiraecola Patch, 1914 (Aphidinae: Aphidini: Aphidina) Planta hospedadora no identificada:

Murcia: Cartagena, sin fecha, Antonio J. Muñoz leg. VALENCIA: Canals, 22-10-2007 y 28-10-2007, Fani Martínez leg.

Aphis verbasci Schrank, 1801 (Aphidinae: Aphidini: Aphidina) Verbascum thapsus:

HuesCA: Benasque, 11-8-2007, Isidro Martínez, leg.

Aploneura lentisci (Passerini, 1856) (Eriosomatinae: Fordini) Pistacia lentiscus:

AlicAnTE: Villena (Sierra Salinas), 10-5-2008, David Molina leg.

Murcia: Cartagena (Rambla del Cañar), 21-8-2007, José Luis Palacios leg.

ZARAGOZA: Perdiguera (Monte Asteruelas), 19-9-2007, Constantino Escuer leg.

Baizongia pistaciae (Linnaeus, 1767) (Eriosomatinae: Fordini) Pistacia terebinthus:

CASTEllón: Sierra de Espadán (Barranco Almanzor), 21-112007, David Molina, leg.

HuesCA*: La Peña, 13-10-2007, Isidro Martínez leg.

JAÉN*: La Iruela, 7-9-2008, Ángel Umaran leg.

TARRAGONA*: L'Espulga de Francolí, 8-9-2007, Jordi Clavell leg.

ZARAGOZA: Mezalocha, 23-8-2008, Isidro Martínez leg.

Brachycaudus amygdalinus (Schouteden, 1905) (Aphidinae: Macrosiphini)

Prunus dulcis:

SORIA*: Alcozar, 20-6-2008, Divina Aparicio leg.

Brachycaudus cardui (Linnaeus, 1758) (Aphidinae: Macrosiphini)

Cynara scolymus:

VALENCIA: Canals, 4-8-2007, 8-8-2007, 28-9-2007 y 1-5-2008, Fani Martínez leg.

Senecio cineraria**:

AlicAnte: Villena (Peña Rubia), 15-6-2008, David Molina, leg.

Planta hospedadora no identificada:

MÁlaga: Málaga, ?-5-2007, Jaime Díaz leg.

Brachycaudus helichrysi (Kaltenbach, 1843) (Aphidinae: Macrosiphini)

Vinca major**:

MADRID: Madrid, 2-4-2008, Ángel Umaran leg.

Brachyunguis tamaricis (Lichtenstein, 1885) (Aphidinae: Aphidini: Aphidina)
Tamarix parviflora:

ZARAGOZA: Zaragoza, 24-9-2007, Isidro Martínez leg.

Tamarix sp.

GERONA*: L'Estartit, 24-6-2008, Jordi Clavell leg.

MADRID: Torrejón de Ardoz, 12-5-2008, Ángel Umaran leg.

Brevicoryne brassicae (Linnaeus, 1758) (Aphidinae: Macrosiphini)

Moricandia arvensis:

ZARAGOZA: Zaragoza, 4-4-2008, Isidro Martínez leg.

Sinapis $\mathrm{sp}$ :

AlicAnTE: Villena, 17-3-2008, David Molina leg.

Brassicaceae:

BARCELONA: L'Hospitalet de Llobregat, 30-4-2008, Divina Aparicio leg.

Planta hospedadora no identificada:

Asturias: Grado, 14-6-2007, Luis M. Lafuente leg.

Ciudad Real*: Puertollano, 14-4-2008, Nacho Cabellos leg.

Capitophorus elaeagni (Del Guercio, 1894) (Aphidinae: Macrosiphini)

Elaeagnus angustifolia:

AlicAnTE: Villena (Peña Rubia), 13-4-2008, David Molina leg.

Cavariella aegopodii (Scopoli, 1763) (Aphidinae: Macrosiphini) Foeniculum vulgare:

Alicante: Villena (Las Virtudes), 16-6-2008, David Molina leg. Petroselinum crispum:

ZARAGOZA: Perdiguera, 9-4-2008, Constantino Escuer leg.

Chaitophorus populialbae (Boyer de Fonscolombe, 1841) (Chaitophorinae: Chaitophorini)

Populus alba:

MADRID: Madrid, 27-6-2008, Ángel Umaran leg.

VALENCIA*: Valencia, 15-11-2007, David Molina leg.

Chromaphis juglandicola (Kaltenbach, 1843) (Calaphidinae: Panaphidini: Panaphidina)

Juglans regia:

AlicAnte: Villena (Peña Rubia), 18-6-2008, David Molina leg. MADRID*: Madrid, 1-9-2008, Ángel Umaran leg.

Dysaphis plantaginea (Passerini, 1860) (Aphidinae: Macrosiphini)

Malus domestica:

MADRID: Madrid, 31-3-2008, Ángel Umaran leg.

Eriosoma lanigerum (Hausmann, 1802) (Eriosomatinae: Eriosomatini)

Malus domestica:

AlmeríA*: Bayárcal (Puerto de la Ragua), 1-7-2007, Miguel Ángel Gómez leg.

MADRID*: Madrid, 28-3-2008 y 14-3-2009, Ángel Umaran leg.

Eriosoma lanuginosum (Hartig, 1839) (Eriosomatinae: Eriosomatini)

Ulmus minor:

AliCANTE: Villena, 25-5-2008; Villena (Huerta de San Juan), 10-4-2005 y 17-6-2007, David Molina leg.

LÉRIDA*: Sant Martí de Maldà, 7-6-2008, Jordi Clavell leg.

VALLADOLID*: Valladolid, 23-6-2007, Juan Carlos Cambronero leg. 
Forda formicaria von Heyden, 1837 (Eriosomatinae: Fordini) Pistacia terebinthus:

Alicante: Villena (Peña Rubia), 13-6-2008, David Molina leg.

HuescA*: Anzánigo, 20-7-2008, Isidro Martínez leg.; La Peña, 13-10-2007, Isidro Martínez leg.

LA RioJa: Torrecilla, 22-9-2007, David Molina leg.

Poaceae (hormiguero):

CASTELlón: Segorbe, 9-1-2008, David Molina leg.

Geoica utricularia (Passerini, 1856) (Eriosomatinae: Fordini) Pistacia terebinthus:

AlicAnte: Villena (Sierra Salinas), 7-9-2008, David Molina leg.

HUESCA*: Anzánigo, 20-7-2008, Isidro Martínez leg.

LA RioJA*: Torrecilla, 22-9-2007, David Molina leg.

Málaga: El Burgo (Mirador del Guarda Forestal), 16-112007, Sue Eatok leg.

VALENCIA: Montesa, 15-8-2008, Fani Martínez leg.

ZARAGOZA: Mezalocha, 23-8-2008, Isidro Martínez leg.

Hoplocallis picta (Ferrari, 1872) (Calaphidinae: Panaphidini: Myzocallidina)

Quercus sp.:

MADRID: Madrid, 11-11-2007, Ángel Umaran leg.

Hyadaphis foeniculi (Passerini, 1860) (Aphidinae: Macrosiphini) Daucus carota:

HuESCA*: Sabiñánigo, 16-9-2007, Isidro Martínez leg.

Hyperomyzus lactucae (Linnaeus, 1758) (Aphidinae: Macrosiphini)

Errante:

MurCiA: Cartagena (La Azohia), 12-4-2007, Joaquín Portela leg.

Kaltenbachiella pallida (Haliday, 1838) (Eriosomatinae: Eriosomatini)

Ulmus minor:

Ávila: Bohoyo, 30-6-2007, Laura Collado leg.

Lachnus roboris (Linnaeus, 1758) (Lachninae: Lachnini)

Quercus coccifera:

TERUEL: Valbona, 28-8-2008, Isidro Martínez leg.

Quercus ilex:

Barcelona: Sant Martí d'Albars, 28-8-2008, Jordi Clavell leg.

Quercus sp.

HuescA: Fonz, 1-11-2007, Javier Cambra leg.

Macrosiphoniella absinthii (Linnaeus, 1758) (Aphidinae: Macrosiphini)

Artemisia absinthium:

AlicANTE*: Villena (Peña Rubia), 20-4-2008, David Molina leg.

Macrosiphoniella artemisiae (Boyer de Fonscolombe, 1841) (Aphidinae: Macrosiphini)

Artemisia absinthium:

SORIA: Alcozar, 21-6-2008, Divina Aparicio leg.

Macrosiphum euphorbiae (Thomas, 1878) (Aphidinae: Macrosiphini)

Oxalis pes-caprae:

MurciA: Cartagena (La Azohia), 30-4-2007, Joaquín Portela leg.
Macrosiphum rosae (Linnaeus, 1758) (Aphidinae: Macrosiphini)

Dipsacus fullomum:

AstuRIAS: Grado, sin fecha, Luis M. Lafuente leg.

Rosa sp.:

BARCELONA: Rubí, 3-12-2007, Tony Romero leg.

GranadA: Pinos Genil, 1-1-2008, Guillermo Booth leg.

LÉRIDA*: Sant Martí de Maldà, 7-6-2008, Jordi Clavell leg.

MADRID: Madrid (Jardines del Campus Sur, Vallecas), 16-32007, 16-2-2008 y 21-2-2008, Ángel Umaran leg.; Collado Villaba, 18-3-2008, José Ramón Correas leg.; Madrid, 23-82007 y 10-4-2008, Miguel Yuste leg.; Leganés, 4-5-2008, Laura Collado leg.

SEGOVIA: Villagonzalo de Coca, 28-6-2008 y 6-8-2008, Miguel Yuste leg.

SORIA: Alcozar, 9-7-2007 y 22-6-2008, Divina Aparicio leg.

TARRAGONA*: Alcover, 2-3-2009, Josep María Solé leg.

ZARAGOZA: Perdiguera, 12-11-2008, Constantino Escuer leg.; Zaragoza, 16-6-2008, Isidro Martínez leg.

Scabiosa maritima:

Alicante: Villena (Peña Rubia), 30-5-2008, David Molina leg.

Planta hospedadora no identificada:

Murcia: El Alamillo (Puerto de Mazarrón), 20-8-2007, José Luis Palacios leg.

Megoura viciae Buckton, 1876 (Aphidinae: Macrosiphini)

Vicia faba:

VALENCIA*: Canals, 21-4-2007, Fani Martínez leg.

Vicia sativa:

LA CoRUÑA*: Betanzos, 30-11-2007, Belén Amarante leg.

Vicia $\mathrm{sp}$ :

Alicante: Villena (Peña Rubia), 15-6-2008, David Molina leg.

Sin planta hospedadora:

GRANADA: Sierra Almijara, 13-4-2008, Javier Gallego leg.

Melanaphis donacis (Passerini, 1862) (Aphidinae: Aphidini: Rhopalosiphina)

Arundo donax:

BARCELONA*: Espluges de Llobregat, 25-11-2007, Divina Aparicio leg.

MurciA*: Cartagena (La Azohia), 8-11-2007, Joaquín Portela leg.

VALENCIA: Valencia, 24-10-2007, David Molina leg.

ZARAGOZA*: Juslibol, 9-9-2007, Isidro Martínez leg.

Melanaphis pyraria (Passerini, 1862) (Aphidinae: Aphidini: Rhopalosiphina)

Piptatherum miliaceum:

ZARAGOZA: Zaragoza, 16-6-2008, Isidro Martínez leg.

Mindarus abietinus Koch, 1857 (Mindarinae)

Abies pinsapo:

MADRID*: Arboreto de la E.T.S.I. de Montes, Universidad Politécnica de Madrid, Ciudad Universitaria, 5-6-2008, Ángel Umaran leg.

Myzocallis carpini (Koch, 1855) (Calaphidinae: Panaphidini: Myzocallidina)

Carpinus betulus:

MADRID: Madrid (Jardines de El Capricho, Alameda de Osuna), 19-10-2008, Ángel Umaran leg. 
Myzocallis castanicola Baker, 1917 (Calaphidinae: Panaphidini: Myzocallidina)

Planta hospedadora no identificada:

MADRID: Madrid, 27-5-2008, Miguel Yuste leg.

Myzocallis coryli (Goeze, 1778) (Calaphidinae: Panaphidini: Myzocallidina)

Corylus avellana:

MADRID: Barajas, 27-9-2008, Ángel Umaran leg.

Myzus persicae (Sulzer, 1776) (Aphidinae: Macrosiphini)

Hibiscus sp.:

MADRID: Madrid, 4-12-2007, Javier Aznar leg.

Solanaceae:

VALENCIA: Bétera, 2-5-2008, Miguel Romá leg.

Planta hospedadora no identificada:

BarcelonA: Barcelona, 24-5-2008, Jordi Clavell leg.; Santa

Fe del Montseny, 10-6-2007, Antonio García leg.

SEviLla: Sevilla, 30-1-2009, Daniel Gutiérrez leg.

Neotoxoptera violae (Pergande, 1900) (Aphidinae: Macrosiphini) Planta hospedadora no identificada:

MADRID*: Madrid, 9-3-2008, Miguel Yuste leg.

Panaphis juglandis (Goeze, 1778) (Calaphidinae: Panaphidini: Panaphidina)

Juglans regia:

ÁlaVA: Villambrosa, 28-7-2008, Carlos Castañeda leg.

HuESCA: Susín, 16-8-2008, Ángel Umaran leg.

MADRID*: Barajas, 15-6-2008, Ángel Umaran leg.

Pemphigus immunis Buckton, 1896 (Eriosomatinae: Pemphigini)

Populus nigra:

ALICANTE: Villena, 1-7-2007, David Molina leg.; Villena (Peña Rubia), 15-6-2008, David Molina leg.

VALENCIA: Valencia, 13-11-2007, David Molina leg.

Pemphigus spyrothecae Passerini, 1860 (Eriosomatinae: Pemphigini)

Populus nigra:

CASTELlón: Soneja, 23-5-2008, David Molina leg.

VALLADOLID*: Valladolid, 20-7-2007, Juan Carlos Cambronero leg.

Phloeomyzus passerinii (Signoret, 1875) (Phloeomyzinae)

Populus alba "Bolleana":

MADRID: Madrid (Jardines del Campus Sur, Vallecas), 26-52007, Ángel Umaran leg.

Phorodon humuli (Schrank, 1801) (Aphidinae: Macrosiphini) Prunus cerasifera:

MADRID: Madrid (Jardines del Campus Sur, Vallecas), 22-52007, Ángel Umaran leg.

Pterochloroides persicae (Cholodkowsky, 1899) (Lachninae: Lachnini)

Citrus limon:

MADRID*: Leganés, 16-6-2008, Laura Collado leg.

Prunus persica:

AlicANTE*: Villena (Peña Rubia), 30-9-2007 y 10-10-2008, David Molina leg.
MurCIA: Monteagudo, 22-6-2008, Klaus Kamppeter leg.

Pterocomma populeum (Kaltenbach, 1843) (Pterocommatinae) Populus nigra:

MADRID: Leganés, 16-6-2008, Laura Collado leg.

Rhopalosiphum maidis (Fitch, 1856) (Aphidinae: Aphidini: Rhopalosiphina)

Zea mays:

MADRID: Valdemanco, 3-11-2007, Max Delgado leg.

Tetraneura caerulescens (Passerini, 1856) (Eriosomatinae: Eriosomatini)

Ulmus minor:

ZARAGOZA*: Peñaflor, 19-5-2007, Isidro Martínez leg.

Tetraneura nigriabdominalis (Sasaki, 1899) (Eriosomatinae: Eriosomatini)

Ulmus minor:

Alicante: Villena, 25-5-2008, David Molina leg.

Ulmus pumila:

MADRID: Madrid (Jardines del Campus Sur, Vallecas), 21-52004, Ángel Umaran leg.

Tetraneura ulmi (Linnaeus, 1758) (Eriosomatinae: Eriosomatini)

Ulmus minor:

AlicAnte: Villena, 25-5-2008, David Molina leg.

LÉRIDA: Sant Martí de Maldà, 7-6-2008, Jordi Clavell leg.

HuESCA: Sallent de Gállego, 7-7-2006, Ángel Umaran leg.

Tinocallis saltans (Nevsky, 1929) (Calaphidinae: Panaphidini: Panaphidina)

Ulmus pumila:

MADRID: Madrid (Jardines del Campus Sur, Vallecas), 28-42008, Ángel Umaran leg.; Torrejón de Ardoz, 26-4-2008, Ángel Umaran leg.

Toxoptera aurantii (Boyer de Fonscolombe, 1841) (Aphidinae: Aphidini: Aphidina)

Citrus limon:

BARCELONA*: La Garriga, 22-2-2009, Jordi Clavell leg.

Tuberolachnus salignus (Gmelin, 1790) (Lachninae: Lachnini) Salix babylonica:

SALAMANCA: Lumbrales, 29-8-2007 y 31-8-2007, Juan Carlos Cambronero leg.

Salix viminalis:

HuesCA: La Peña, 14-10-2007, Isidro Martínez leg.

Salix sp.:

Burgos*: Villasur de Herreros, 20-8-2008, Jacinto Benhadi leg.

CASTELlón: Segorbe, 31-10-2007, David Molina leg.

Uroleucon sonchi (Linnaeus, 1767) (Aphidinae: Macrosiphini) Sonchus oleraceus:

Alicante: Villena (Peña Rubia), 9-10-2007, David Molina leg. Sonchus tenerrinus:

MurciA*: Totana, 5-5-2005, José Luis Palacios leg

Sonchus sp.:

CASTEllón: Villareal, 27-4-2008, Román Rojo leg.

VALENCIA: Canals, 16-3-2008, Fani Martínez leg. 
Wahlgreniella arbuti (Davidson, 1910) (Aphidinae: Macrosiphini) Arbutus unedo:

AlicAnTE*: Villena (Peña Rubia), 13-6-2008, David Molina leg.

MADRID: Madrid (Jardines del Campus Sur, Vallecas), 29-22008, Ángel Umaran leg.; Madrid (Parque del Retiro), 27-22008, Ángel Umaran leg.

Wahlgreniella nervata (Gillette, 1908) (Aphidinae: Macrosiphini) Rosal sp.:

Huelva*: Zalamea La Real, 17-3-2008, Juan Antonio Perea leg. 